\title{
R-parity violating chargino decays at the LHC
}

\author{
N.-E. Bomark, ${ }^{a}$ A. Kvellestad, ${ }^{b}$ S. Lola, ${ }^{c}$ P. Osland ${ }^{d}$ and A.R. Raklev ${ }^{b, e}$ \\ ${ }^{a}$ National Centre for Nuclear Research, \\ Hoza 69, 00-681 Warsaw, Poland \\ ${ }^{b}$ Department of Physics, University of Oslo, \\ P.O. Box 1048, Blindern, N-0316 Oslo, Norway \\ ${ }^{c}$ Department of Physics, University of Patras, \\ GR-26500 Patras, Greece \\ ${ }^{d}$ Department of Physics and Technology, University of Bergen, \\ P.O. Box 7803, N-5020 Bergen, Norway \\ ${ }^{e}$ Department of Applied Mathematics and Theoretical Physics, University of Cambridge, \\ Wilberforce Road, Cambridge CB3 OWA, U.K. \\ E-mail: nilserik.bomark@gmail.com, anders.kvellestad@fys.uio.no, \\ magda.lola@cern.ch, per.osland@ift.uib.no, ahye@fys.uio.no
}

AbSTRACT: Supersymmetric models with R-parity violation (RPV) have become more popular following the lack of any excess of missing energy events at the $8 \mathrm{TeV}$ LHC. To identify such models, the suggested searches generally rely on the decay products of the (effectively) lightest supersymmetric particle (LSP), with signals that depend on the identity of the LSP and the relevant RPV operators. Here we look at the prospects for detecting RPV chargino decays at the LHC and find substantial patches of parameter space in the Minimal Supersymmetric Standard Model with possibly spectacular signatures, such as three charged-lepton resonances.

KeYwords: Supersymmetry Phenomenology

ArXiv EPRINT: 1410.0921 


\section{Contents}

1 Introduction 1

2 Neutralino-chargino mass difference $\quad 3$

3 Parameter scan 4

3.1 Scan set-up 4

$\begin{array}{ll}3.2 & \text { Results of scan }\end{array}$

4 Implications for collider searches $\quad 8$

4.1 RPV chargino decays and current bounds $\quad 8$

$\begin{array}{lll}4.2 & \text { Displaced vertices from chargino decays } & 10\end{array}$

$\begin{array}{lll}4.3 & \text { LHC resonance searches } & 13\end{array}$

$\begin{array}{llr}5 & \text { Conclusions } & 18\end{array}$

\section{Introduction}

Most searches for supersymmetry with R-parity violation (RPV), see e.g. [1] for a review, adopt one of the following approaches: either one looks for the effects of the relevant operators at low energies in precision measurements, or one focuses on the decay of the (effectively) lightest supersymmetric particle (LSP) through RPV operators. This is the result of the strict bounds on most such couplings, which imply that RPV decay widths are typically subdominant, unless they are the only viable option. For the same reason, possibly spectacular signatures, such as resonant single superparticle productions, are only viable for a limited number of operators and for a constrained range of couplings. In most cases, the LSP, with no other alternative decays available, is the best candidate to look at.

The plethora of RPV operators implies that there is a broad range of potential signals [1], which also depend on the nature of the LSP. In the presence of RPV, any sparticle may in principle be the LSP, since bounds on stable massive charged particles no longer apply. However, the lightest chargino is not usually considered, because common lore says that the structure of the gaugino mass matrices is such that the lightest neutralino is always lighter than the lightest chargino.

In this paper we point out that this is not necessarily true. As discussed by Kribs et al. [2], in the general Minimal Supersymmetric Standard Model (MSSM) the chargino could in principle be lighter than the lightest neutralino in a corner of the parameter space. Even when heavier, the small mass difference that is possible and even natural in certain scenarios, implies that the chargino, as the next-to-lightest sparticle (NLSP), can have dominant decays through RPV operators. 
Models with almost-degenerate electroweak gauginos, where such chargino decays can be expected, arise for example in the context of anomaly-mediated supersymmetry breaking $[3,4]$. The current absence of any supersymmetry (SUSY) signal at the LHC, and the question of the naturalness of the remaining parameter space, has led to the consideration of so-called Natural SUSY models. Here only the higgsinos, the stops, the left-handed sbottom, and, to a more limited extent, the gluino, are light enough to be probed at the LHC $[5,6]$. Higgsino dominance of light neutralinos and charginos also leads to small mass differences, although we have recently shown that, given current direct and indirect constraints, the degeneracy is considerably less severe than for winos [7].

In R-parity conserving (RPC) models, if the degeneracy is severe enough, such spectra can lead to rather characteristic experimental signals in high-energy collisions, with charginos that live long enough to create displaced vertices, or even pass through the detector before decaying [8-10]. Here we instead study the consequences of lifting the RPC. In particular, we are here interested in the trilinear RPV operators in the superpotential:

$$
W \sim \lambda_{i j k} L_{i} L_{j} \bar{E}_{k}+\lambda_{i j k}^{\prime} L_{i} Q_{j} \bar{D}_{k}+\lambda_{i j k}^{\prime \prime} \bar{U}_{i} \bar{D}_{j} \bar{D}_{k}
$$

We do not include bilinear RPV operators of the form $\mu_{i} H_{u} L_{i}$. These will induce mixing between the charginos and the charged leptons, and allow decays for the lightest chargino of the form $\tilde{\chi}_{1}^{ \pm} \rightarrow \nu_{i} W^{ \pm}, l_{i}^{ \pm} Z$. These decays have already been discussed for AMSB scenarios motivated by neutrino masses in [11].

Direct chargino decays via subsets of RPV operators have been considered in the past, especially in the context of LEP physics, see e.g. [12]. Nevertheless, a detailed discussion that also takes into account the rich flavour structure of the RPV operators is still lacking. Here, we investigate the consequences of direct RPV chargino decays in the context of LHC searches, and we show that this can lead to dramatic signals, such as resonant three-lepton final states due to $L L \bar{E}$ operators. In addition, the presence of heavy quarks in $L Q \bar{D}$ and $\bar{U} \bar{D} \bar{D}$ operators results in enhanced detection prospects in this case as well.

One may object to breaking R-parity, since it ensures the existence of a stable sparticle, realising one of the central motivations for weak-scale supersymmetry: the existence of dark matter. However, as pointed out in recent years [13-15], the gravitino may be the real LSP, with a naturally long lifetime, due to its tiny gravitational coupling. For a wide range of parameters gravitinos are essentially stable on cosmological time-scales and can act as dark matter. Despite this, we will refer to the neutralino and chargino as the effective LSP to avoid a very convoluted language. ${ }^{1}$

In this paper, we present an update on how near-degenerate electroweak gauginos arise in general supersymmetry breaking models described by the MSSM, and to what extent they are compatible with the recent discovery of a new boson at the LHC, when interpreted as the light SM-like Higgs state of the MSSM [16, 17], and other direct and indirect constraints. We study the possibility of a light chargino with the MultiNest $2.17[18,19]$ code for parameter sampling, which enables a detailed analysis of the posterior probability

\footnotetext{
${ }^{1}$ Note also that this is not compatible with the standard anomaly-mediated breaking scenario, where the gravitino is heavy compared to the other sparticles, which have loop-suppressed masses.
} 
distribution in the supersymmetric parameter space of interest, taking into account the available experimental constraints. We then study the effect of RPV operators on these models in the context of searches at the LHC.

We should note that the interesting properties of chargino decays that we find within our scenario, also apply to extensions of the theory beyond the MSSM. In this respect direct chargino decays can be a powerful tool to probe the gaugino sector and distinguish between different possibilities. Significant deviations from gaugino unification can arise naturally in well-motivated scenarios, for instance due to the presence of F-terms [20, 21]; in such schemes, the specific gaugino hierarchies to be expected are fixed to a large extent by the group theory, and the argument can be reversed: if direct chargino decays via RPV operators are detected at significant rates, we will have crucial information on the structure of the gaugino sector and on the underlying GUT symmetries of the theory.

We begin in section 2 by discussing the parameters that affect the neutralino-chargino mass difference in the MSSM. We then describe the parameter scan that we have performed in section 3. In section 4.1 we look at the consequences of current bounds on the RPV couplings and competition with the RPC chargino decays. In section 4.2 we disucss the impact of current LHC searches for displaced vertices on our scenario. Finally, we describe the consequences of our results for LHC searches for RPV chargino decays in section 4.3, before we conclude in section 5 .

\section{Neutralino-chargino mass difference}

In the MSSM, the free mass parameters in the neutralino mass matrix at tree level are $M_{1}, M_{2}$ and $\mu$. In addition, $\tan \beta$ also enters as a free parameter. With the exception of $M_{1}$, the same set of parameters enters in the tree-level chargino mass matrix. Any complex phases for the mass parameters are very constrained, in particular due to limits on the electric dipole moments [22-25]. However, there are no a priori grounds not to give arbitrary signs to these parameters, although by a rotation of basis we can choose $M_{2}$ to always be positive.

For small $M_{1}$ the lightest neutralino will be a bino, which is historically the most popular choice. Since $M_{1}$ does not enter in the chargino mass matrix, in this case there is no degeneracy between chargino and neutralino. When $\mu$ or $M_{2}$ is the smallest parameter we may have a neutralino that is dominantly a higgsino or a wino, and in both cases there may be degeneracy with the chargino.

In the wino limit, $M_{2}<\left|M_{1}\right|, \mu$, the tree-level mass difference

$$
\Delta m \equiv m_{\tilde{\chi}_{1}^{ \pm}}-m_{\tilde{\chi}_{1}^{0}}
$$

expanded in $1 / \mu$ is $[9,10]$

$$
\begin{aligned}
\Delta m= & \frac{M_{W}^{2}}{\mu^{2}} \frac{M_{W}^{2}}{M_{1}-M_{2}} \tan ^{2} \theta_{W} \sin ^{2} 2 \beta+2 \frac{M_{W}^{4} M_{2} \sin 2 \beta}{\left(M_{1}-M_{2}\right) \mu^{3}} \tan ^{2} \theta_{W} \\
& +\frac{M_{W}^{6} \sin ^{3} 2 \beta}{\left(M_{1}-M_{2}\right)^{2} \mu^{3}} \tan ^{2} \theta_{W}\left(\tan ^{2} \theta_{W}-1\right)+\mathcal{O}\left(\frac{1}{\mu^{4}}\right) .
\end{aligned}
$$


Note that while this can give a negative $\Delta m$ for negative $M_{1}$, these tree-level terms are all small for large $\tan \beta$, and for $\tan \beta \rightarrow \infty$ the lowest contributing order is in fact $1 / \mu^{4}$. This means that loop effects can be significant. The leading loop correction from gauge bosons - assuming there are no very light sfermions - is positive and in the wino limit is given by $[9,26]$

$$
\Delta m_{1-\text { loop }}=\frac{\alpha_{2} M_{2}}{4 \pi}\left[f\left(M_{W} / M_{2}\right)-\cos ^{2} \theta_{W} f\left(M_{Z} / M_{2}\right)-\sin ^{2} \theta_{W} f(0)\right],
$$

where

$$
f(a)=2 \int_{0}^{1}(1+x) \ln \left(x^{2}+(1-x) a^{2}\right) d x .
$$

In the limit where $M_{2} \gg M_{W}$, this gives $\Delta m_{1-\text { loop }} \simeq 165 \mathrm{MeV} .^{2}$ The possibility of getting a mass difference $\lesssim 165 \mathrm{MeV}$ then rests on the contribution from eq. (2.2) being negative and significant compared to the expression (2.3). This could be the case for negative $M_{1}$.

In the higgsino limit, $\left|M_{1}\right|, M_{2}>\mu, M_{W}$, the tree-level mass difference from an expansion in $1 / M_{2}$ is [27]:

$$
\Delta m=\left[\frac{M_{2}}{M_{1}} \tan ^{2} \theta_{W}+1+\operatorname{sgn} \mu\left(\frac{M_{2}}{M_{1}} \tan ^{2} \theta_{W}-1\right) \sin 2 \beta\right] \frac{M_{W}^{2}}{2 M_{2}}+\mathcal{O}\left(\frac{1}{M_{2}^{2}}\right) .
$$

This expansion breaks down for $\mu \rightarrow 0$, however, LEP limits on the chargino mass ensure that we can keep out of that region of parameter space.

For positive $M_{1}$ and $M_{2}, \Delta m$ in the higgsino limit is always positive. It becomes small for very large $M_{1}, M_{2} \gg M_{W}$, but numerically this does not lower the mass difference below $300 \mathrm{MeV}$, unless (i) both masses are greater than $\mathcal{O}(10 \mathrm{TeV})$, or (ii) $\tan \beta \simeq 1$ and either mass is very large. For a negative $M_{1}$, however, we may have a negative $\Delta m$, but this occurs for very special choices of the parameters, namely: relatively small $\left|M_{1}\right|$, combined with large $M_{2}$ and small $\tan \beta$, see [7]. In addition to the above, we have loop corrections that mainly stem from top-stop and $\gamma(Z)$-higgsino loops. The former can have either sign depending on the stop mixing, while the latter is small unless $\tan \beta$ is large. Both are included in the scan that will be performed in the next section.

\section{Parameter scan}

\subsection{Scan set-up}

To search for these degenerate models, we employ a bayesian scan over the MSSM parameter space, using the three parameters $M_{1}, M_{2}$ and $M_{3}$ to represent the gaugino mass for the $\mathrm{U}(1), \mathrm{SU}(2)$ and $\mathrm{SU}(3)$ sectors, respectively, at the electroweak scale. $M_{1}$ and $M_{3}$ are allowed to take negative values. For the Higgs sector we use the parameters $\mu, m_{A^{0}}$ and $\tan \beta$, deriving $m_{H_{u}}$ and $m_{H_{d}}$ from EWSB. These are the relevant parameters for the problem at hand. Furthermore, we use a common mass parameter $m_{\tilde{q}}$ for the first and second

\footnotetext{
${ }^{2}$ Note that, apart from a numerical factor, this electroweak correction is, as expected, $\alpha M_{W}$, which just happens to be of the order of the pion mass.
} 


\begin{tabular}{|lccc|}
\hline Parameter & Range & Prior & Reference \\
\hline$M_{1}$ & {$[-4000,4000]$} & $\log$ & - \\
$M_{2}$ & {$[0,4000]$} & $\log$ & - \\
$M_{3}$ & {$[-4000,4000]$} & $\log$ & - \\
$\mu$ & {$[-4000,4000]$} & $\log$ & - \\
$m_{A^{0}}$ & {$[0,4000]$} & $\log$ & - \\
$m_{\tilde{l}}$ & {$[0,7000]$} & $\log$ & - \\
$m_{\tilde{q}}$ & {$[0,7000]$} & $\log$ & - \\
$m_{\tilde{q}}$ & {$[0,7000]$} & $\log$ & - \\
$A_{0}$ & {$[-7000,7000]$} & $\log$ & - \\
$\tan \beta$ & {$[2,60]$} & $\operatorname{linear}$ & - \\
\hline$m_{t}$ & $173.4 \pm 1.0$ & gaussian & {$[29]$} \\
$m_{b}^{\overline{M S}}\left(m_{b}\right)$ & $4.18 \pm 0.03$ & gaussian & {$[30]$} \\
$M_{Z}$ & $91.1876 \pm 0.0021$ & gaussian & {$[30]$} \\
$\alpha^{-1}$ & $127.944 \pm 0.014$ & gaussian & {$[30]$} \\
$\alpha_{s}$ & $0.1184 \pm 0.0007$ & gaussian & {$[30]$} \\
\hline
\end{tabular}

Table 1. List of scan parameters with ranges and priors. Dimensionful parameters are given in $\mathrm{GeV}$. All non-SM parameters are given at the scale $Q=1.0 \mathrm{TeV}$, except $\tan \beta$ and $\mu$ which are given at the EWSB scale, and the pseudoscalar Higgs pole mass $m_{A^{0}}$. Log priors are set to zero over the ranges $(-25,25)$ and $(0,25) \mathrm{GeV}$ for signed and non-negative parameters, respectively.

generation squarks, while a separate parameter $m_{\tilde{q}_{3}}$ is used for squarks of the third generation. Finally, the sleptons are governed by a common mass scale $m_{\tilde{l}}$, and we use a common value $A_{0}$ for the trilinear couplings. We do not scan over the RPV couplings directly, as the sheer number of couplings makes this unfeasible. However, in section 4 we discuss in detail the allowed values of these couplings, and study how they affect the chargino lifetime and branching fractions for the set of posterior samples produced by the scan.

For all mass parameters we use logarithmic priors in order to incorporate a prior belief in naturalness [28], while for $\tan \beta$ a flat prior is used. The SM parameters $m_{t}, m_{b}, M_{Z}$, $\alpha$ and $\alpha_{s}$ are included as nuisance parameters with gaussian priors. A summary of the parameters used, along with ranges and priors, is given in table 1. For comparison, we also perform a scan with flat priors for all parameters except the SM nuisance parameters. This scan is performed with less statistics compared to the main scan, as it is only used to check the prior dependence of our results.

The scan uses MultiNest $2.17[18,19]$ to explore the parameter space described above. For each point in the parameter space, the sparticle spectrum is calculated by SoftSusy 3.3.5 [31], including the effects discussed in section 2 for the chargino-neutralino mass difference, while Higgs masses are calculated using FeynHiggs 2.9.4 [32-35]. We apply constraints from electroweak precision observables and B-physics, using SoftSusy and MicrOMEGAS 2.4.5 [36-38] to calculate the relevant quantities. In addition, the relevant 


\begin{tabular}{|lccc|}
\hline Observable & Constraint & Likelihood & Reference/Comment \\
\hline$M_{W}$ & $80.385 \pm 0.021$ & gaussian & {$[42]$} \\
$a_{\mu}^{\exp }-a_{\mu}^{\mathrm{SM}}$ & $(26.1 \pm 8.0) \times 10^{-10}$ & gaussian & {$[43,44]$} \\
$\mathrm{BR}\left(B_{s} \rightarrow \mu \mu\right)$ & $2.9_{-1.0}^{+1.1} \times 10^{-9}$ & from experiment & {$[40,41]$} \\
$\mathrm{BR}(b \rightarrow s \gamma)$ & $(3.55 \pm 0.33) \times 10^{-4}$ & gaussian & {$[45]$} \\
$\mathrm{R}(B \rightarrow \tau \nu)$ & $1.63 \pm 0.54$ & gaussian & {$[45]$} \\
$m_{h}$ & $125.0 \pm 2.0$ & gaussian & {$[46]$} \\
$m_{\tilde{\chi}_{1}^{ \pm}}$ & $>45$ & lower limit, hard cut & {$[47]$} \\
$m_{\tilde{\chi}_{1}^{ \pm}}-m_{\tilde{\chi}_{1}^{0}}$ & $<1.0$ & upper limit, hard cut & see text \\
\hline
\end{tabular}

Table 2. List of the constraints used in the full likelihood for the scans. All masses are given in $\mathrm{GeV}$. Experimental and theoretical errors have been added in quadrature.

constraints from LEP data on the chargino mass and the LHC Higgs mass measurement are included. We note that most limits on the chargino mass from LEP are void due to the small $\Delta m$. The values and distributions used for these constraints are summarised in table 2 . Note that although the CMS limit on $\mathrm{BR}\left(B_{s} \rightarrow \mu \mu\right)$ [39], is slightly more constraining than the corresponding LHCb limit [40,41], since the latter provides a likelihood covering a wider range of branching ratio values, we use that in our scan.

No dark matter constraints have been applied; with R-parity violation the most natural dark matter candidates would be gravitinos or axions, thus the standard WIMP relic abundance, direct and indirect detection constraints do not apply. Nor have we applied constraints from direct LHC searches for coloured sparticles. These can be avoided by pushing all the squark masses and the gluino mass up, albeit at the price of a loss of naturalness. Even the most restrictive of these bounds (for squark and gluino masses) affect the chargino-neutralino mass difference only through small loop corrections. Direct limits on chargino-neutralino production depend intimately on the RPV coupling in question, and will be discussed in section 4 .

As a check, scans have also been performed with a modified version of the public code SuperBayeS 1.5.1 [48, 49], where the relevant quantities are calculated by SoftSusy ${ }^{3}$ and DarkSusy 5.0 [50]. The conclusions from these scans agree very well with the ones reached with the setup described above.

In order to focus the scan on light charginos, we also demand a chargino or neutralino LSP and impose an upper limit on the chargino-neutralino mass difference. These constraints on the likelihood, which are not from observables, are in effect restricting us to a subset of the MSSM parameter space, a model where the lightest chargino and neutralino have a small mass difference and one of them is the LSP. Despite being the result of a somewhat convoluted definition, there is in principle nothing that sets this model apart at the electroweak scale from other constrained models based on the MSSM field content, e.g. mSUGRA; the constraints are only different.

\footnotetext{
${ }^{3}$ Due to problems with loop contributions to the neutralino and chargino masses in SoftSusy 2.0.18, which is the version included in SuperBayes, we have updated its SoftSusy version to 3.3.7.
} 

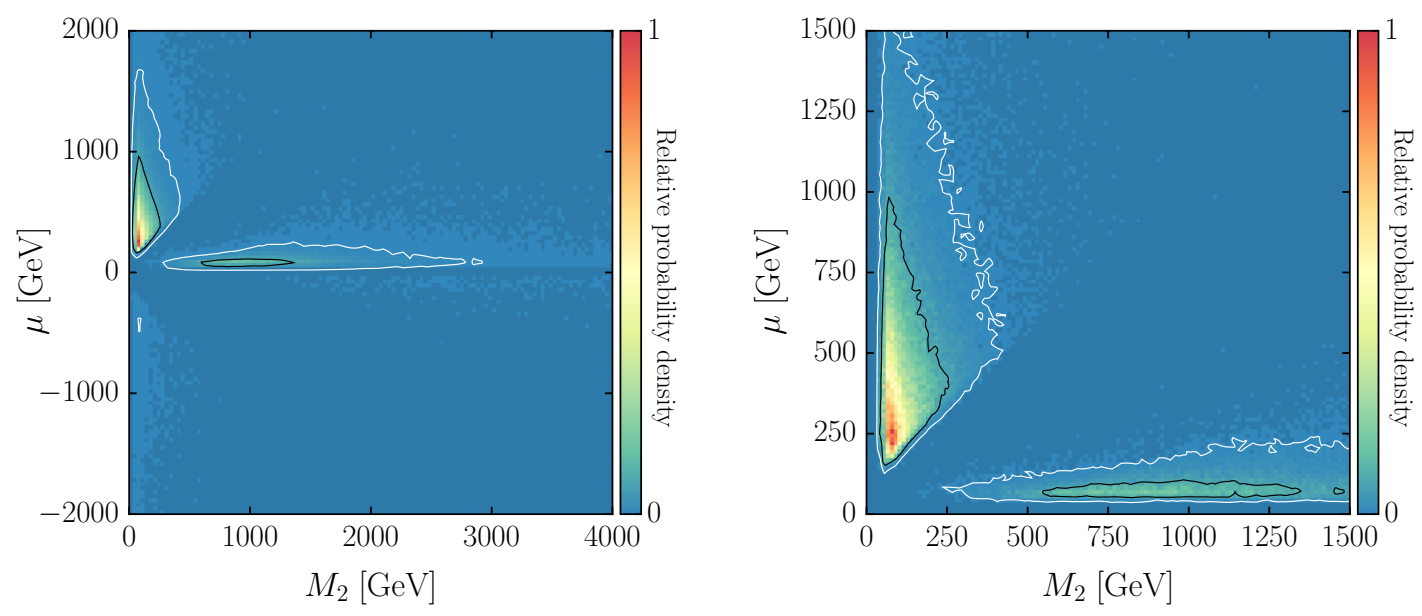

Figure 1. Marginalized posterior in the $M_{2}-\mu$ plane. The $68 \%$ and $95 \%$ C.R. contours are shown in black and white, respectively.

\subsection{Results of scan}

In figure 1 we show the marginalised posterior probability distribution in the $M_{2}-\mu$ plane (left), with a higher resolution plot for smaller parameter values (right). The colour scale represents the magnitude of the probability distribution relative to its maximum point and contours of the $68 \%$ and $95 \%$ credible regions (C.R.) are shown in black and white, respectively. We see in principle four distinct areas, two each with wino and higgsino LSP but with different sign of $\mu$. However, the $g-2$ requirement for the muon results in a preference for a positive value of $\mu$ (same sign as $M_{2}$ ), and the resulting area of parameter space with negative $\mu$ is very small and outside the $68 \%$ and $95 \%$ C.R. contours, except for a tiny area with a wino LSP ( $\operatorname{small} M_{2}$ ).

From the scan it is clear that a wino LSP is preferred in the MSSM, $M_{2}<|\mu|$, when we restrict ourselves to models with small $\Delta m$. This is a consequence of the general difficulty in achieving a small mass difference from eq. (2.5) for the higgsinos at tree level, made worse by the relatively high Higgs mass that favours large $\tan \beta$. In addition, adding the constraint on the anomalous magnetic moment of the muon, further favours small $M_{2}$. We pointed out a very similar situation in the MSSM restricted to Natural SUSY models in [7].

In figure 2 (left) we show the marginalised posterior distribution in the $m_{\tilde{\chi}_{1}^{0}}-\Delta m$ plane. We see that the points naturally accumulate around the $150 \mathrm{MeV}$ mass difference given by the wino radiative correction of order $\alpha_{2} M_{2} / 4 \pi$. However, there is still a significant part of the preferred parameter space that has negative mass difference. To compare the wino and higgsino cases, in figure 2 (right), we plot only the posterior points with a higgsino LSP.

The preference for a wino scenario, $M_{2}<|\mu|$, is even stronger in the scan using flat priors. Following the shift in priors, the posterior distributions for the mass parameters are weighted towards higher absolute values. This increases the importance of the wino radiative correction in eq. (2.3), thus strengthening the preference for $\Delta m$ values around $150 \mathrm{MeV}$. Also, the range of preferred chargino and neutralino masses is widened, with the $68 \%$ and $95 \%$ C.R. in the $m_{\tilde{\chi}_{1}^{0}}-\Delta m$ plane extending up to $m_{\tilde{\chi}_{1}^{0}} \sim 650 \mathrm{GeV}$ and $m_{\tilde{\chi}_{1}^{0}} \sim 1100 \mathrm{GeV}$, respectively, for $\Delta m \sim 165 \mathrm{MeV}$. 

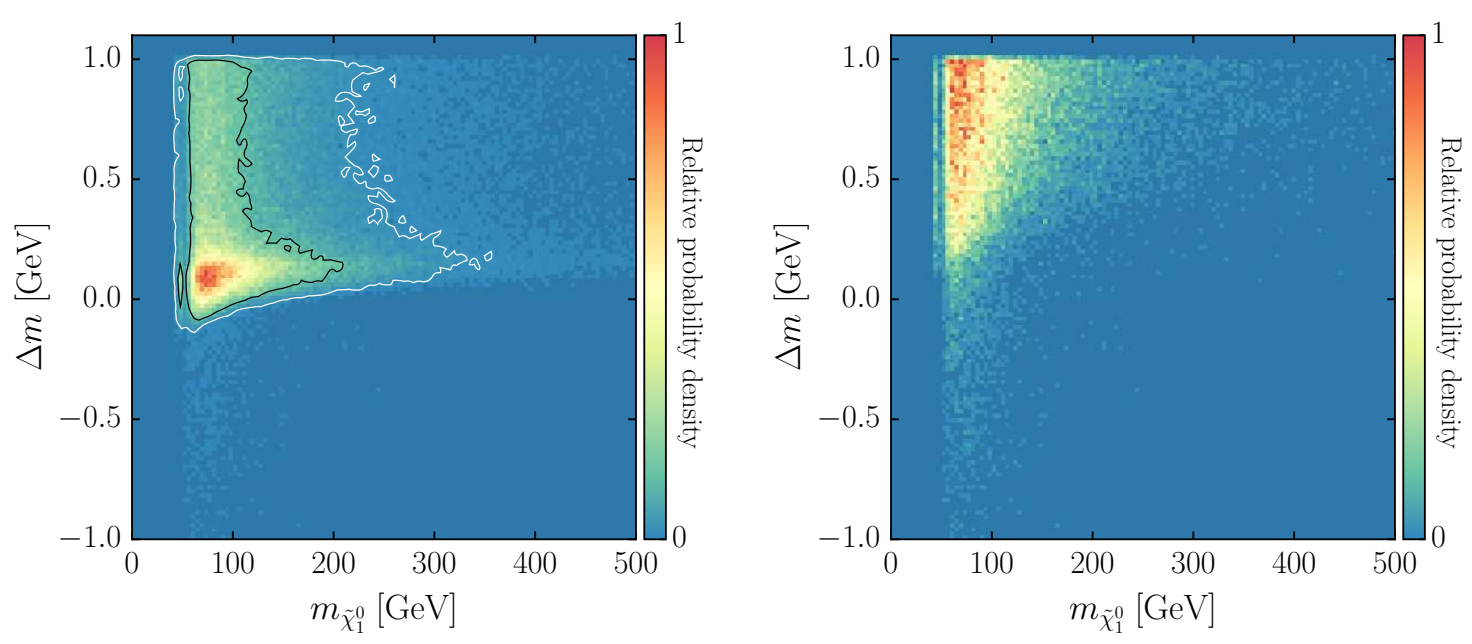

Figure 2. Marginalized posterior in $m_{\tilde{\chi}_{1}^{0}}$ versus $\Delta m$ for all neutralinos (left) and for higgsino-like neutralinos only (right). The $68 \%$ and $95 \%$ C.R. contours are shown in black and white, respectively.

\section{Implications for collider searches}

The propensity for a mass difference $\Delta m \sim m_{\pi^{ \pm}}$in the degenerate scenario means that in R-parity conserving models the relevant decay modes of the chargino are $\tilde{\chi}_{1}^{ \pm} \rightarrow \tilde{\chi}_{1}^{0}\left(e^{ \pm} \nu, \mu^{ \pm} \nu\right)$ and $\tilde{\chi}_{1}^{ \pm} \rightarrow \tilde{\chi}_{1}^{0} \pi^{ \pm}$, where the latter is dominant [8]. If R-parity is violated we must also consider the three-body decays of the chargino to three fermions via a virtual sfermion. Depending on the size and flavour of the RPV couplings, and to some extent the sfermion masses, as well as $\Delta m$, this might instead be the dominant decay channel.

Here we discuss the implication of RPV chargino decays for collider searches, starting by describing the parameter space where these are dominant and the consequences of current bounds on the RPV couplings. Then, we continue with a discussion of the possibility of displaced vertices and the limits that can be set from the absence of massive metastable particles at the LHC. Finally, we turn to the possibility of direct searches for chargino resonances at the $13 \mathrm{TeV} \mathrm{LHC}$.

\subsection{RPV chargino decays and current bounds}

If the mass difference between the chargino and the neutralino is small but still larger than $m_{\pi^{ \pm}}$, the most important R-parity conserving decay channel for the chargino is $\tilde{\chi}_{1}^{ \pm} \rightarrow \tilde{\chi}_{1}^{0} \pi^{ \pm}$. The decay width of this channel has been given in [8]. The competing R-parity violating decay widths were given in $[12,51]$.

We begin by studying the effect of the $L L \bar{E}$ operators on our set of posterior samples. Figure 3 shows the resulting posterior distribution in the planes of relevant chargino branching ratios versus the mass difference $\Delta m$. Here we have assumed a dominant RPV coupling of $\lambda_{121}$. The current best experimental limit on this coupling, from chargedcurrent universality, is at the weak scale $\lambda_{121}<0.049 \times \frac{m_{\tilde{e}_{R}}}{100 \mathrm{GeV}}[52,53]$. In the discussion below we take the upper bounds for all couplings from [53]. For each posterior point we choose the largest allowed value of the coupling. We remind the reader that the effect of changing the size of the coupling is a simple scaling of the RPV widths as $\lambda^{2}$. 

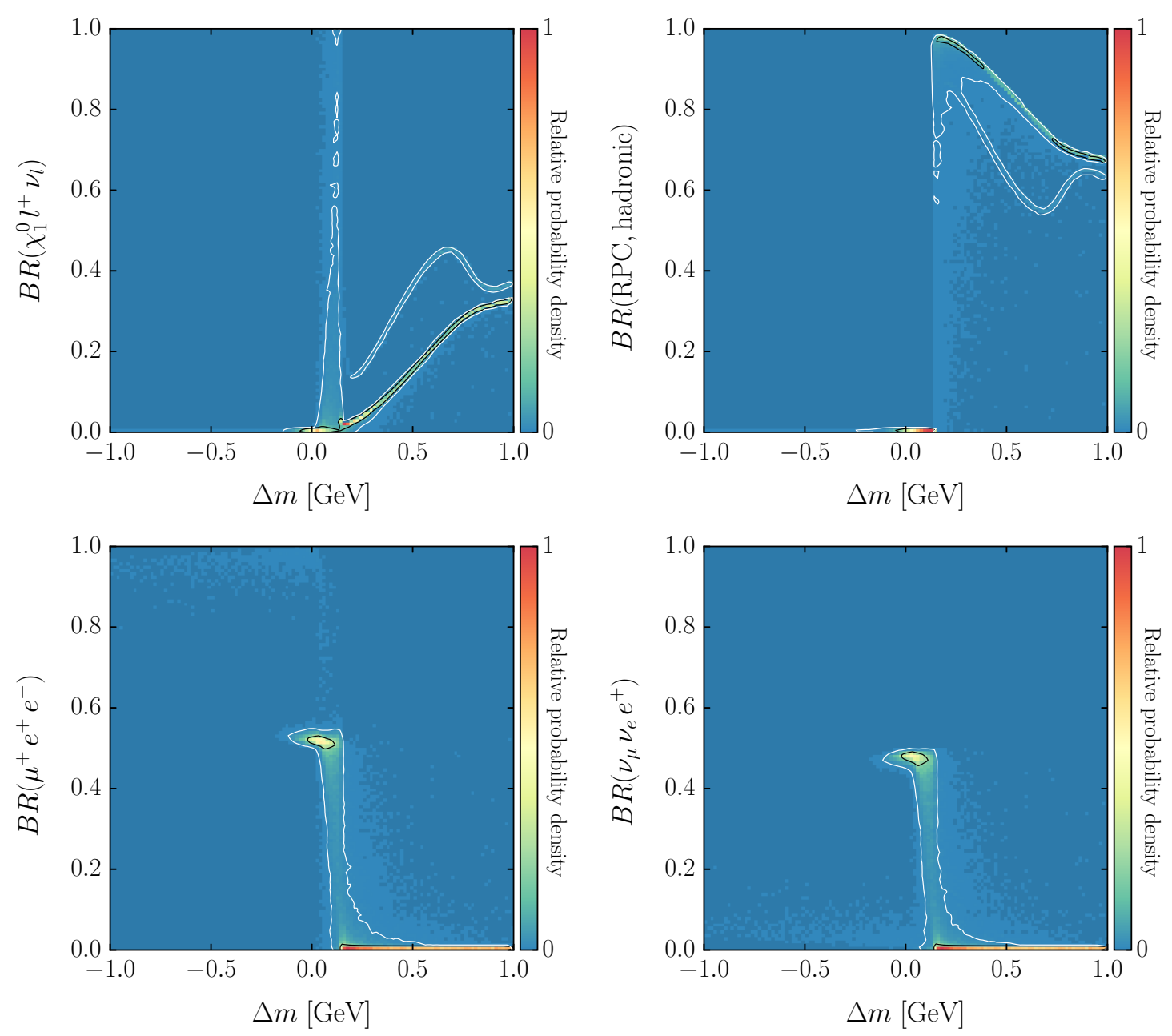

Figure 3. Posterior distribution of $\Delta m$ versus the branching ratio for various chargino decay channels. The $68 \%$ and $95 \%$ C.R. contours are shown in black and white, respectively.

The top panels of figure 3 show the RPC decays to leptons (left) and hadrons (right). The latter dominate down to mass differences of $\sim 0.15 \mathrm{GeV}$, near the pion threshold. The two-pronged structure of these plots in the $95 \%$ C.R. contour shows the difference between a wino and higgsino LSP, where the higgsinos prefer leptonic decays. We note that, given the discovery of a long-lived chargino with a displaced vertex in the detector, the nature of the decay products could potentially be used to discriminate between wino and higgsino.

The RPV decays shown in the lower panels of figure 3 demonstrate that below the pion threshold there is a roughly equal splitting between the decay to three charged leptons, $e^{+} \mu^{+} e^{-}$, and the decay to two neutrinos $\nu_{e} \nu_{\mu} e^{+}$, with the three charged leptons slightly dominant. The other allowed modes, to $\bar{\nu}_{e} \mu^{+} \nu_{e}$ and $e^{+} \bar{\nu}_{\mu} \nu_{e}$, both require the propagator to come from the $\bar{E}$ field which, due to its right chirality, does not couple to winos, while the coupling to higgsinos is suppressed by the small electron mass. This equitable distribution ends when the chargino becomes sufficiently light, at which point the decay to three charged leptons alone is completely dominant. This is a consequence of these charginos being 
higgsinos, and therefore the decay to three charged leptons is governed by the muon Yukawa coupling while the decay to two neutrinos comes from the electron Yukawa. Note though, that this scenario is outside of the $95 \%$ C.R. contour of the posterior distribution.

The decay pattern we see here has important phenomenological consequences since it is relatively easy to reconstruct the chargino from three charged leptons.

Our initial choice of value for $\lambda$ maximized the RPV effect, however, lowering $\lambda$ will affect branching ratios only in the region between the pion mass threshold and $\Delta m=0$ where RPC and RPV processes compete. Here we find, fixing the sfermion mass and changing the RPV coupling, that the total RPV decays on average ${ }^{4}$ reach a branching ratio of $\sim 0.5$, when $\lambda_{121} \sim 0.05$, compared to $\sim 1$ when $\lambda_{121} \sim 1$. For much lower values of $\lambda_{121}$ the RPC decay is completely dominant. The scaling with the sfermion mass, from the sfermion propagator in the RPV decays, is $m_{\tilde{f}}^{-4}$. Thus an increase of the sfermion mass of a factor 4.5 has the same effect as a reduction in the coupling from 1 to 0.05 . Note also that the RPV width goes as $m_{\tilde{\chi}_{1}^{ \pm}}^{5}$ while the RPC decay only depends on $\Delta m$, so for higher chargino masses the RPV decay will tend to be more dominant.

When flat priors are used, the posterior probability for having sizeable branching ratios for RPV decays increases slightly. This is mainly due to the increased probability for small $\Delta m$ values resulting from stronger wino dominance. Also, the above-mentioned dependence of the RPV widths on $m_{\tilde{\chi}_{1}^{ \pm}}$and $m_{\tilde{f}}$ becomes more important as the range of probable values for these masses widens when using flat priors. The net result of this is a preference for RPV widths that are typically larger by a factor of a few compared to the scan with log priors.

Very similar results are found for the remaining RPV couplings. Changing the fermion masses changes the higgsino coupling. However, using for example $\lambda_{323}$ (the operator with the heaviest leptons allowed by $\mathrm{SU}(2)$ invariance) we find only negligible differences with respect to the branching ratio distributions.

Turning to the $L Q \bar{D}$ and $\bar{U} \bar{D} \bar{D}$ operators we find that $L Q \bar{D}$ sees the same behaviour as $L L \bar{E}$, with an equipartition of RPV branching ratios below the pion threshold between the final states $\ell_{i}^{+} d_{j} \bar{d}_{k}$ and $\nu_{i} u_{j} \bar{d}_{k}$. The exception to this is $L_{i} Q_{3} \bar{D}_{k}$ where the phase space suppression of the final state top quark implies that $\ell_{i}^{+} b \bar{d}_{k}$ is dominant; the remaining parameter space where the chargino is heavy enough to easily decay to an on-shell top quark is small, see figure 2.

For $\bar{U} \bar{D} \bar{D}$ the generally heavy squark propagators, needed for the high Higgs mass, suppresses the RPV decays resulting in dominant RPC decays down to mass differences of $\Delta m \simeq 0.01 \mathrm{GeV}$ even for $\lambda^{\prime \prime}=1$, and results in much longer chargino lifetimes when $\Delta m \in\left[10 \mathrm{MeV}, m_{\pi}\right]$. More on this below.

\subsection{Displaced vertices from chargino decays}

In the region $\Delta m<m_{\pi}$, where RPV processes can dominate, the parameters $\lambda$ and $m_{\tilde{f}}$ will influence the lifetime of the chargino and it is interesting to ask whether such models can give rise to detectable displaced vertices from late chargino decays.

\footnotetext{
${ }^{4}$ Marginalized over the posterior sample.
} 

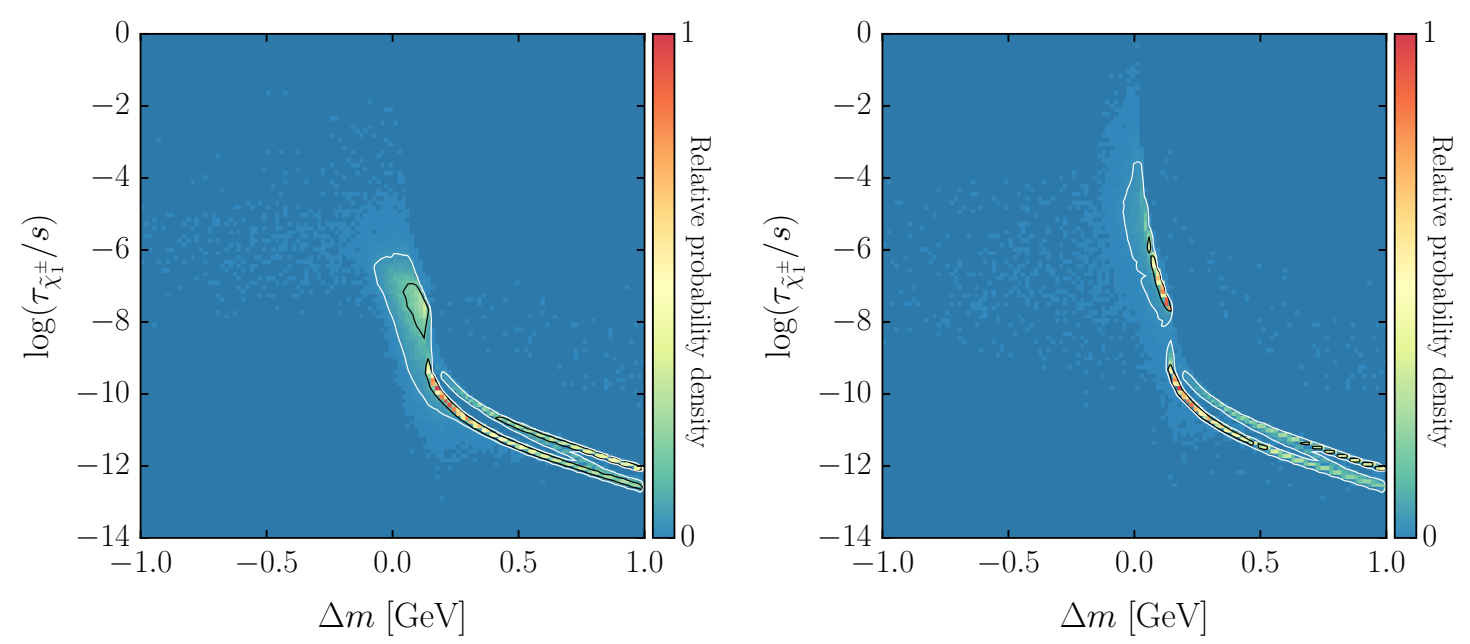

Figure 4. The posterior distribution of $\Delta m$ versus chargino lifetime with the $L_{1} L_{2} \bar{E}_{1}$ coupling $\lambda_{121}$ (left) and the $\bar{U}_{3} \bar{D}_{2} \bar{D}_{3}$ coupling $\lambda_{323}^{\prime \prime}$ (right). The $68 \%$ and $95 \%$ C.R. contours are shown in black and white, respectively.

In figure 4 we show the posterior distribution in the plane of chargino lifetime and $\Delta m$, with the same assuptions on the RPV coupling as above. ${ }^{5}$ We see that for the $\lambda_{121}$ coupling and low mass differences (left figure) lifetimes of $10^{-11}-10^{-7} \mathrm{~s}$ are within the $68 \%$ C.R. contours. For the $\lambda_{323}^{\prime \prime}$ coupling (right figure) the dominance of RPC decays mentioned above drives the lifetime up even further. The visible break is the change in RPC decays from pions to leptons at $\Delta m=m_{\pi}$. Compared to the results in figure 4 , the scan with flat priors prefers lifetimes shorter by a factor of a few in the region $\Delta m \lesssim 200 \mathrm{MeV}$, corresponding to the previously noted shift towards larger RPV widths.

Values of $c \tau>1 \mathrm{~cm}$ or $\tau>3.3 \times 10^{-11} \mathrm{~s}$, will give rise to a substantial number of kinked tracks in the inner detector of for example ATLAS [54], and should be detectable in the LHC experiments if a sufficient number of charginos are produced. Recent searches in ATLAS have set limits down to lifetimes of $0.06 \mathrm{~ns}(c \tau=1.8 \mathrm{~cm})$ [55] in AMSB models where the chargino is dominantly wino. We have checked our posterior sample against these limits, assuming a dominant $\lambda_{121}$ coupling, and find that in the conservative interpretation, where we assume that the charginos have a pure wino production cross section, the competitive region $\Delta m \in\left[0, m_{\pi}\right]$ is moderated, excluding parts of the parameter space with lifetime longer than $0.1 \mathrm{~ns}$ and chargino masses below $150 \mathrm{GeV}$.

Nevertheless, significant parts of the parameter space remain within the $68 \%$ C.R. with a $50 \%$ branching ratio to three charged leptons. This surviving region prefers somewhat heavier charginos than the posterior sample following the scan, relatively high $\tan \beta$, and sees lifetimes in the region $0.1-0.01 \mathrm{~ns}$, which can be within the future reach of the LHC experiments looking for displaced vertices. We study three such points, RPV_C1, RPV_C2 and RPV_C3, as benchmark points in the next section. Details of these points are given in table 3 .

\footnotetext{
${ }^{5}$ The limit used for $\lambda_{323}^{\prime \prime}$ is the perturbative limit [53] and is not dependent on the sfermion masses.
} 


\begin{tabular}{|lccc|}
\hline Point & RPV_C1 & RPV_C2 & RPV_C3 \\
\hline$m_{\tilde{\chi}_{1}^{ \pm}}$ & 252.1 & 327.7 & 526.4 \\
$\Delta m$ & 0.119 & 0.108 & 0.182 \\
Wino & 0.990 & 0.986 & 0.989 \\
Higgsino & 0.142 & 0.166 & 0.148 \\
\hline$M_{1}$ & 944.1 & -1082.0 & -728.4 \\
$M_{2}$ & 235.4 & 311.4 & 502.3 \\
$M_{3}$ & 1627.6 & 560.6 & 3418.6 \\
$\mu$ & 668.0 & 668.5 & 913.2 \\
$m_{A^{0}}$ & 3430.3 & 2775.5 & 3220.5 \\
$m_{\tilde{l}}$ & 503.5 & 434.6 & 757.6 \\
$m_{\tilde{q}}$ & 2156.2 & 2517.0 & 4742.9 \\
$m_{\tilde{q}_{3}}$ & 6429.4 & 4951.8 & 1424.6 \\
$A_{0}$ & -25.8 & 2775.5 & 1498.1 \\
$\tan \beta$ & 47.1 & 55.4 & 46.2 \\
\hline
\end{tabular}

Table 3. Summary of the properties of the benchmark points studied. All masses are in GeV.

The dependence of these conclusions on which RPV coupling is dominant, for a particular class of operators, say $L L \bar{E}$, is very weak because of the assumptions in the scan, namely that of common weak-scale sfermion soft masses. For the $L Q \bar{D}$ operators the situation is similar to $L L \bar{E}$, with somewhat shorter lifetimes predicted. This is because the dominant RPV decay channels, $\ell_{i}^{+} d_{j} \bar{d}_{k}$ and $\nu_{i} u_{j} \bar{d}_{k}$, have the same slepton propagators as the dominant $L L \bar{E}$ decays; at the same time the upper bound on the coupling size is less restrictive, since, for most couplings, this depends on large squark masses. After applying the ATLAS limits from displaced vertices, we still have significant regions of the parameter space with dominant decays to $\ell_{i}^{+} d_{j} \bar{d}_{k}$ and $\nu_{i} u_{j} \bar{d}_{k}$ within the $68 \%$ confidence region. One exception here is $\lambda_{111}^{\prime}$ where the bound on the coupling from neutrino-less double beta decay is sufficiently strong to exclude most points with dominant RPV decays, except points that also have very large slepton masses.

For $\bar{U} \bar{D} \bar{D}$ the heavy squark propagators reduce the decay width for RPV decays, leading to longer chargino lifetimes as compared to $L L \bar{E}$ and $L Q \bar{D}$. As a result, the displaced vertices search excludes all regions of the preferred parameter space, the $95 \%$ C.R., where RPV decays from $\bar{U} \bar{D} \bar{D}$ are substantial, having above $1 \%$ branching ratio.

The restrictions on the individual RPV couplings, from above by the indirect bounds taken from [53], and from below by the ATLAS lifetime bounds, when seen together, are quite severe for all the preferred regions in the parameter space discussed in section 3 . As an example we list maximum values for the $L L \bar{E}$ couplings in table 4 for the benchmark points RPV_C1, RPV_C2 and RPV_C3. The minimum values for RPV_C1 vary in the range $0.222-$ 0.228. Since the ATLAS bound extends up to chargino masses of $500 \mathrm{GeV}$, it is difficult to find points with low fine-tuning that completely escape this bound. For comparison, a benchmark point RPV_C2 with higher chargino mass has minimum values in the range 


\begin{tabular}{|lccccccccc|}
\hline Point/Coupling & $\lambda_{121}$ & $\lambda_{122}$ & $\lambda_{123}$ & $\lambda_{131}$ & $\lambda_{132}$ & $\lambda_{133}$ & $\lambda_{231}$ & $\lambda_{232}$ & $\lambda_{233}$ \\
\hline RPV_C1 & 0.244 & 0.244 & 0.260 & 0.309 & 0.309 & 0.013 & 0.349 & 0.349 & 0.372 \\
\hline RPV_C2 & 0.215 & 0.215 & 0.221 & 0.272 & 0.272 & 0.011 & 0.307 & 0.308 & 0.316 \\
\hline RPV_C3 & 0.369 & 0.369 & 0.388 & 0.467 & 0.467 & 0.016 & 0.527 & 0.528 & 0.554 \\
\hline
\end{tabular}

Table 4. Maximum allowed values of the $L L \bar{E}$ couplings for the benchmark points discussed in the text.

0.055-0.059, where the less severe bounds are in part also caused by lower slepton masses. The point RPV_C3 has no such lower bound, since it has a chargino mass above $500 \mathrm{GeV}$ and is thus outside the reach of the ATLAS search. From these results, we conclude that certain combinations of couplings and points with dominant RPV decays are unlikely in the context of natural models; in particular a dominant $\lambda_{133}$ coupling is hard to realize.

\subsection{LHC resonance searches}

To study the possibility of observing RPV chargino decays at the LHC we generate events at $13 \mathrm{TeV}$ using PYTHIA 8.1.80 [57], with FASTJET 3.0.6 [58] for jet reconstruction using the $k_{t}$-algorithm $[59,60]$ with jet radius $R=0.4 .^{6}$ We use the single dominant coupling approximation. ${ }^{7}$ As the search for RPV decays of charginos is similar to the corresponding decays of neutralinos, we refer to [61] for details on the analysis. Here we will mostly focus on the differences that appear when charginos are involved.

$\boldsymbol{L} \boldsymbol{L} \overline{\boldsymbol{E}}$ operators. The most obvious difference between neutralinos and charginos, as discussed in the previous section, is that for $L L \bar{E}$ operators, the chargino has a large branching ratio for $\tilde{\chi}_{1}^{ \pm} \rightarrow l l l, l \nu \nu$ via a virtual sneutrino or slepton, raising the possibility to observe a resonance of three charged leptons. Since for $\lambda_{i j k} \mathrm{SU}(2)$ invariance requires $i \neq j$, there are always at least two different flavours in the final state leptons. Thus, the pure leptonic combinations that are most relevant for collider searches are $e^{+} e^{-} \mu^{+}\left(\lambda_{121}\right)$, and $\mu^{+} \mu^{-} e^{+}\left(\lambda_{122}\right)$. The remaining $L L \bar{E}$ operators have some tau flavour in them, which necessarily smears out any resonance peak.

We employ the cuts used in [61], which require many high $p_{T}$ leptons and missing energy:

- Three isolated leptons with $p_{T}>70,20,20 \mathrm{GeV}$ within the detector's geometric acceptance.

- Missing transverse energy $E_{T}^{\text {miss }}>100 \mathrm{GeV}$.

We simulate LHC data for benchmark points RPV_C1 and RPV_C3 taken from our scan, with the largest possible value of the RPV couplings allowed for the slepton masses of those points. These couplings were given in table $4 .{ }^{8}$ A full PYTHIA simulation of neutralino

\footnotetext{
${ }^{6}$ In order to calculate decay widths and branching rations for the sparticles, including RPV operators, we use PYTHIA 6.4.25 [56], modified to include the $\tilde{\chi}_{1}^{ \pm} \rightarrow \tilde{\chi}_{1}^{0} \pi^{ \pm}$decay.

${ }^{7}$ We note that large R-violating hierarchies can be expected in many models, similarly to the large hierarchies in the Yukawa couplings that generate fermion masses.

${ }^{8}$ Since for our benchmark points the RPV decay is clearly dominating, relatively small changes to $\lambda$ will not affect the result, larger changes would decrease the signal as $\lambda^{2}$.
} 
and chargino pair production is performed, meaning that both the lightest neutralino and chargino will decay through the RPV couplings. The presented signal distributions are therefore a superposition stemming from both types of decays.

In figure 5 we show the resulting tri-lepton invariant mass distributions for the benchmark point RPV_C3 with the $L_{1} L_{2} \bar{E}_{1}, L_{1} L_{2} \bar{E}_{3}, L_{2} L_{3} \bar{E}_{2}$ and $L_{1} L_{3} \bar{E}_{3}$ operators (from top left to bottom right) for signal and dominant background, ${ }^{9}$ normalized to $1 \mathrm{fb}^{-1}$ of integrated luminosity. The $\tau$ in these plots refers to hadronic jets stemming from taus. As expected, $L_{1} L_{2} \bar{E}_{1}$ shows a clearly identifiable peak at the chargino mass of $526 \mathrm{GeV}$ above the combinatorial background. For $L_{1} L_{2} \bar{E}_{3}$ and $L_{2} L_{3} \bar{E}_{2}$ we see clearly identifiable features in the distributions with taus as well as identifiable kinks in the purely leptonic distributions. For these couplings the chargino should be observable as a resonance up to quite high masses at $13-14 \mathrm{TeV}$. Due to the large content of taus, $L_{1} L_{3} \bar{E}_{3}$ appears more challenging; there is a small kink in the $e \tau \tau$ distribution, but one should bear in mind that these plots are produced from $10^{6}$ generated events (before cuts) and this is an unrealistically high number as compared to experimental expectations, so it remains an open question whether these smaller features can actually be observed.

From preliminary studies by ATLAS of the tri-lepton reach in supersymmetry models at $14 \mathrm{TeV}$ [62], the main background to the tri-lepton resonance is expected to be diboson production, in the absence of a $Z$-boson veto, and tri-boson and $t \bar{t}$ with said veto. Comparing the total NLO cross section of $175 \mathrm{pb}$ for di-boson production at $13 \mathrm{TeV}$ [63], to the total neutralino and chargino pair-production cross section for RPV_C1 and RPV_C3 of $0.99 \mathrm{pb}$ and $49.9 \mathrm{fb}$, respectively, calculated using Prospino 2.1 [64], both benchmark points seem promising for early discovery. ${ }^{10}$

In order to quantify this further, we can use the selection efficiency of the di-boson background and signal for the $e e \mu$ final state under the cuts given above, ${ }^{11}$ which we find to be $5.7 \times 10^{-5}$ for the background, and 0.24 and 0.47 for RPV_C1 and RPV_C3, respectively. ${ }^{12}$ Using an $S / \sqrt{B}$ criterion to test sensitivity, where $S$ is the number of signal events and $B$ the background, and requiring a minimum of five signal events, a simple event counting experiment will need $21 \mathrm{pb}^{-1}$ and $0.45 \mathrm{fb}^{-1}$ of data to reach discovery level for the two benchmarks. This is clearly an early discovery opportunity for Run II of the LHC. Because of the very low SM background, in order to observe the chargino peak above the combinatorics of the events with supersymmetric origin, slightly more statistics would be needed. Results for the $e \mu \mu$ state are very similar.

More detailed studies could be performed by the LHC experiments, to take into account the missing energy resolution and the lepton reconstruction efficiency, which should be high

\footnotetext{
${ }^{9}$ For the tri-lepton distributions we use WZ production for the background (see below for a discussion of the backgrounds) while for the distributions including tau-jets we use ZZ production since WZ will not give tau-jets in addition to the three leptons required to pass the cuts.

${ }^{10}$ We note here that some of the four-lepton searches in [65] may be sensitive to chargino masses as low as in RPV_C1, however, it is difficult to fully understand their impact without a detailed simulation of each search, which is outside the scope of the present paper. RPV_C3 should be safely outside of their reach.

${ }^{11}$ In order to explore the many possible RPV couplings we have kept our cuts very generic. Improvements for specific couplings can certainly be made. However, we feel a more detailed study of cuts and sensitivity, than our rough estimate, would be better done within the experiments.

${ }^{12}$ Calculated using leading order matrix elements in the Monte Carlo simulation.
} 

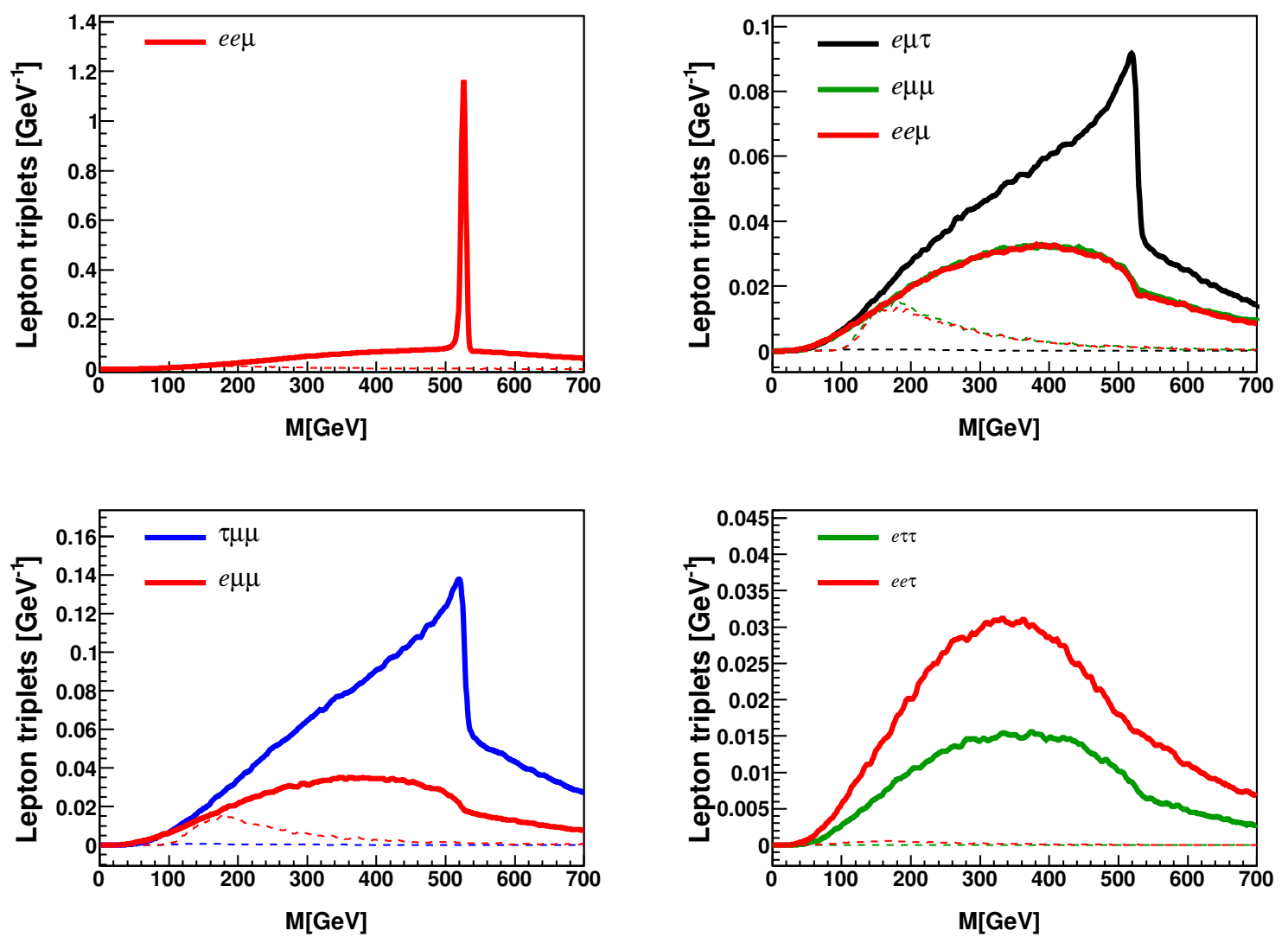

Figure 5. Various flavour combinations of tri-lepton invariant masses for the $L_{1} L_{2} \bar{E}_{1}, L_{1} L_{2} \bar{E}_{3}$, $L_{2} L_{3} \bar{E}_{2}$ and $L_{1} L_{3} \bar{E}_{3}$ couplings. The thin dashed lines give the dominant background and the distributions are normalized to $1 \mathrm{fb}^{-1}$ of integrated luminosity.

at the transverse momenta of interest, and to include a full background analysis. Here we took into account only the most significant background. These however go beyond the scope of this paper.

Looking for the tri-lepton peak is crucial for identifying the scenario giving rise to a multi-lepton excess. It would tell us that we have a charged particle that decays to a specific combination of three leptons, thus violating lepton number, as well as the mass of that particle, and potentially the spin of the particle through angular distributions of the decay products. As already mentioned, the distributions of figure 5 may help with these identifications also for those operators that do not give rise to a clear peak.

As in the case of neutralino decays discussed in [61], there are also interesting features in the di-lepton invariant mass distributions. The advantage with di-lepton distributions is that we can employ same-sign subtraction to practically remove the combinatorial background and hence reveal features otherwise invisible. The combinatorial background consists of lepton pairs that come from different parts of the event and are hence expected to be uncorrelated. As a result, the charges of these lepton pairs should be uncorrelated and therefore, taking for example the difference, $m_{e^{+} e^{-}}-m_{e^{-} e^{-}}-m_{e^{+} e^{+}}$for the ee invariant mass, should remove the combinatorial background. 

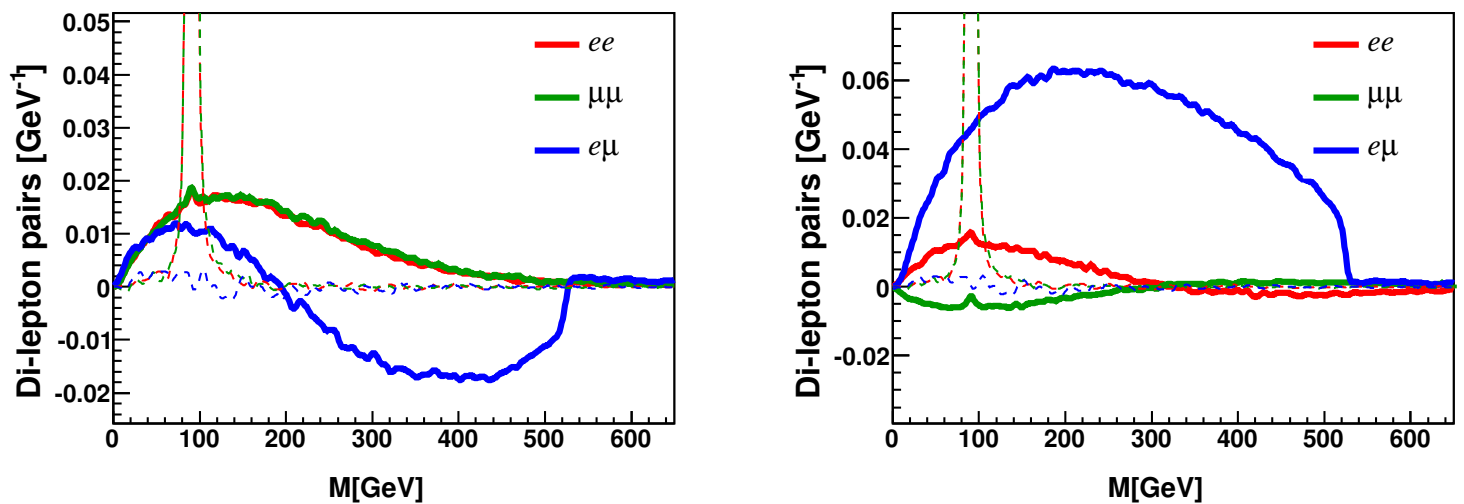

Figure 6. Di-lepton invariant masses for $L_{1} L_{2} \bar{E}_{3}$ (left) and $L_{2} L_{3} \bar{E}_{1}$ (right). The thin dashed lines give the dominant background and the distributions are normalized to $1 \mathrm{fb}^{-1}$ of integrated luminosity.

As can be seen in figure 6, where the resulting di-lepton invariant mass distributions for $L_{1} L_{2} \bar{E}_{3}$ and $L_{2} L_{3} \bar{E}_{1}$ (signal and WZ background for $1 \mathrm{fb}^{-1}$ of integrated luminosity) are shown, this works as expected. For neutralino decays, these distributions were discussed in detail in [61]; here we focus on those features that are relevant for decaying charginos. The most striking feature can be seen in the left panel of figure 6 where the $e \mu$ invariant mass distribution becomes negative. This effect is due to the structure of the couplings; the two distinct flavours in the lepton superfield doublets $L$ will give rise to contributions in the same-sign invariant mass distributions from the chargino decays. After same-sign subtraction this will show up as a negative contribution in the distribution, and interfere with the positive contributions from both decaying charginos and neutralinos.

Following [61], the cleanness of the di-lepton distributions can be used to extract a substantial amount of information about the scenario at hand, including the chargino/neutralino mass and the flavours of the couplings, also in the difficult cases where the decays are dominated by taus. In addition one could hope to establish the ratio of neutralino to chargino decays in the final distribution.

To illustrate this, let us look at the $e e$ and $\mu \mu$ distributions in the right-hand panel of figure 6 . The negative perturbation in the $\mu \mu$ channel shows that we have a decaying chargino, while the absence of a clear cutoff as in the $e \mu$ channel indicates that one of the muons comes from a decaying tau. This means that our coupling must have an $L_{2} L_{3}$ component. The large peak in the $e \mu$ channel indicates that the last component of the RPV operator must be $\bar{E}_{1}$, and it also gives us the chargino/neutralino mass. Finally, the positive perturbation in the ee distribution is caused by neutralino and chargino decays with an $e e$ pair where one of the electrons comes from a decaying tau and the other from the $\bar{E}_{1}$ operator.

We can now compare the height of the perturbation in the ee distribution to the one of the negative perturbation in the $\mu \mu$ distribution. Since the contribution from chargino decays is the same in both distributions, the difference in height between the positive and negative perturbations, when compared to the (negative) height of the $\mu \mu$ distribution, 

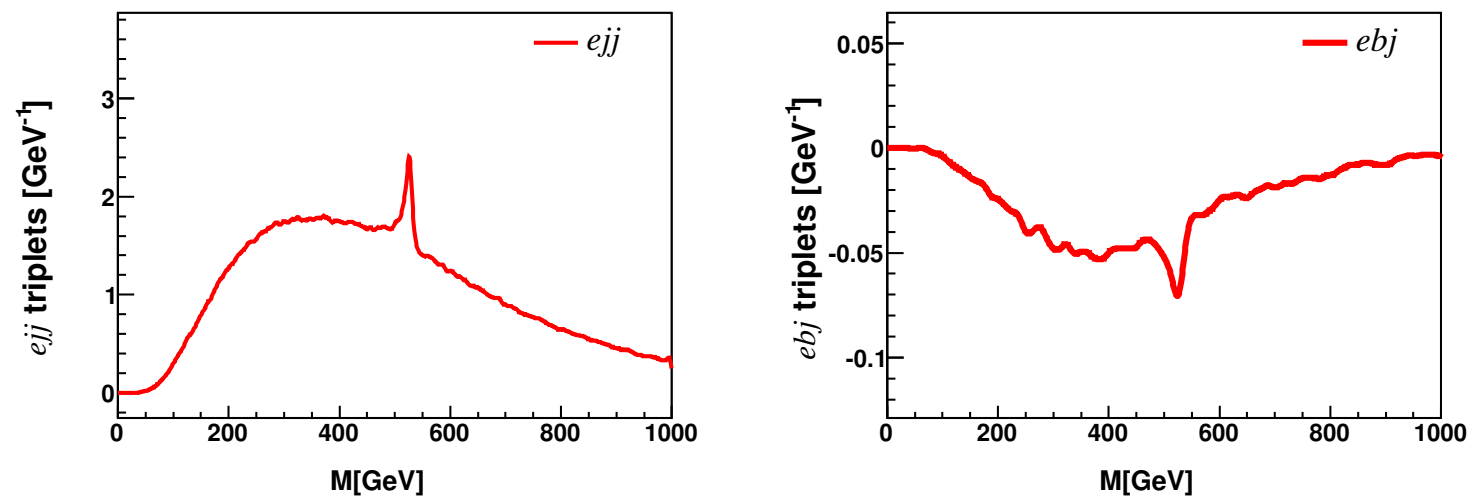

Figure 7. Lepton plus dijet invariant mass distributions for $L_{1} Q_{1} \bar{D}_{1}$ (left), and the same for $L_{1} Q_{3} \bar{D}_{1}$ including same-sign subtraction (right). Signal only, normalized to $1 \mathrm{fb}^{-1}$ of integrated luminosity.

reveals the ratio of neutralino to chargino decays in our event sample. In this case charginos are slightly more common than neutralinos.

$\boldsymbol{L} \boldsymbol{Q} \overline{\mathbf{D}}$ operators. In figure 7 (left) we show the distribution of the invariant mass of $l j j$ combinations for the RPV_C3 benchmark point with the $L_{1} Q_{1} \bar{D}_{1}$ operator for signal only, with arbitrary normalization. Since light quark flavour, and thus charge, is impossible to determine experimentally from jet physics, chargino resonances decaying through the $L Q \bar{D}$ operators have already received significant attention in searches for leptoquarks and neutralinos with RPV decays. We will therefore limit our discussion here. In terms of chargino masses the same cross section limits should apply as for neutralinos.

One exception that is worth mentioning are operators of the type $L_{i} Q_{3} \bar{D}_{k}$ that cause relatively light neutralinos to always decay to neutrino plus jets, $\nu_{i} b \bar{d}_{k}$, while heavier neutralinos can decay to $l_{i}^{-} t \bar{d}_{k}$. Light charginos will always have the possibility to decay to charged lepton plus jets, $l_{i}^{-} b \bar{d}_{k}$, thus improving the detectability significantly with both a lepton and a b-tag, and potential charge identification, for the resonance reconstruction. This can be further improved by same-sign subtraction for the electron and b-jet pair in order to reduce combinatorial background. We show the resulting distribution of

$$
m_{e^{-\bar{b} j}}+m_{e^{+} b j}-m_{e^{+} \bar{b} j}-m_{e^{-} b j},
$$

for the $L_{1} Q_{3} \bar{D}_{1}$ operator in figure 7 (right). Here the chargino contribution has been subtracted, showing up as the downward fluctuation in the distribution at $\sim 530 \mathrm{GeV}$.

We also note that models with large $\lambda_{111}^{\prime}$ and $m_{\tilde{\chi}_{1}^{0}}$ from $500 \mathrm{GeV}$ to $1 \mathrm{TeV}$ were recently studied [66] as a possible explanation of the deviations from the Standard Model seen in the eejj channel in CMS data [67], however, this requires sleptons with masses around $2 \mathrm{TeV}$, which are very disfavoured in our search due to the naturalness bias and the constraint on $(g-2)_{\mu}$ that we have applied. Even so, it is worth pointing out that a degenerate chargino-neutralino pair is not impossible in this scenario, and that such a chargino can be searched for as a resonance in the $l^{+} d \bar{d}$ channel. 
$\overline{\boldsymbol{U}} \overline{\boldsymbol{D}} \overline{\boldsymbol{D}}$ operators. For the $\bar{U} \bar{D} \bar{D}$ operators the situation is similar to the $L Q \bar{D}$ operators. The chargino decays as $\tilde{\chi}_{1}^{+} \rightarrow u_{i} u_{j} d_{k}, \bar{d}_{i} \bar{d}_{j} \bar{d}_{k}$. For light flavours a chargino resonance will look just like a neutralino (or gluino) resonance decaying into three quarks, $\tilde{\chi}_{1}^{0} \rightarrow$ $u_{i} d_{j} d_{k}$, and will be equally challenging to reconstruct, although some improvement could be had from the sophisticated use of jet clustering algorithms [68]. Experimental bounds on three-jet resonances do exist [69], but these have been interpreted only in terms of gluino production and RPV decay, where the production cross sections are much higher. As we have seen in section 4.2, RPV decays of charginos through $\bar{U} \bar{D} \bar{D}$ operators should be heavily suppressed compared to RPC decays in scenarios that feature naturalness, and will thus be even more difficult to discover.

We end by pointing out that for the operators $\bar{U}_{3} \bar{D}_{j} \bar{D}_{k}$, a neutralino decay requires a top quark in the final state, while the chargino can also decay to three down quarks, of which at least one is a bottom quark. As discussed above in section 4.2 it seems difficult to realise a scenario where the chargino is much lighter than $500 \mathrm{GeV}$ and decays dominantly through RPV couplings. Thus, this inherent difference may have only marginal applicability since the decay to top quarks will not be very kinematically suppressed at such high masses. The final states unique to the chargino are the decays to two b-quarks and one light quark, with the experimental signature $b b j$, and the decays to two top quarks and a light down quark, $t t j$, which are possible for the $\bar{U}_{3} \bar{D}_{1} \bar{D}_{3}$ and $\bar{U}_{3} \bar{D}_{2} \bar{D}_{3}$ operators.

\section{Conclusions}

Following the lack of missing-energy signals at the LHC, there is now additional motivation to depart from the MSSM and study in more detail alternative realisations of supersymmetry. A promising scenario in this respect is provided by R-parity violation, where missing energy is substituted by multi-lepton or multi-jet events. While especially the latter can often be hard to distinguish from the SM backgrounds, there are channels where the signals can be spectacular. Further motivation for such schemes is provided by the fact that R-parity violation can be perfectly compatible with supersymmetric dark matter, with a gravitino LSP that is stable on cosmological scales, and rapid decays of the remaining superparticles inside the detector, thus being detectable.

Within this framework, we previously studied neutralino decays at the LHC, paying particular attention to the rich flavour structure of the theory. Here, we extend these studies to direct RPV chargino decays, which can take place in scenarios with a wino or higgsino effective LSP, where the lightest chargino and neutralino have a small mass difference $\Delta m$. In order to investigate the properties of these scenarios, we employ a bayesian scan over the MSSM parameter space, taking into account the relevant constraints. We perform our main scan with logarithmic priors, checking that the central conclusions remain unchanged with linear priors. In addition to concrete experimental signatures related to RPV chargino decays, we also identify distinct differences between a wino and a higgsino LSP. For instance the first is somewhat preferred in the region of small $\Delta m$, while the second has a larger $\mathrm{RPC}$ branching ratio to leptons, which, if a long-lived chargino is discovered, can be used to probe the gaugino sector of the theory. 
For the RPV decays we find the following interesting possibilities:

- For $L L \bar{E}$ operators, spectacular signatures, such as three charged-lepton resonances, with explicit lepton number violation in the final state, can be expected. We have shown that this can be the case for substantial patches of the supersymmetric parameter space, even in the MSSM, which is the most conservative scenario. In extensions of the theory, the parameter space where such signatures could arise would be enhanced. In addition, similarly to neutralino decays, there are interesting features in the di-lepton invariant mass distributions, where employing same-sign subtraction practically removes the combinatorial background, thus revealing features that would otherwise be invisible.

- For $L Q \bar{D}$ operators, the expected physics is similar to RPV neutralino decays, but with some quite interesting differences. Particularly for $L Q_{3} \bar{D}$, there is a distinct difference between RPV neutralino and chargino decays, since the first involves neutrinos plus jets or a charged lepton and a top quark, while the latter involves charged leptons plus jets or neutrinos and top quarks. For charginos therefore, a signal with both a lepton and a b-tag and potential charge identification has enhanced detectability.

- For $\bar{U} \bar{D} \bar{D}$ operators we do not expect significant chargino RPV decays for positive $\Delta m$. For small (below pion mass) and negative mass differences charginos below $500 \mathrm{GeV}$ seem excluded by searches for long-lived charged particles. For heavier charginos detection through RPV decays seems very difficult, the use of sophisticated jet clustering methods is in general required. However, we note that the operators $\bar{U}_{3} \bar{D}_{j} \bar{D}_{k}$ have a particularly interesting behaviour: while neutralino RPV decays will always contain a top quark, the chargino can decay to three jets including at least one b-jet. Furthermore, for the operators $\bar{U}_{3} \bar{D}_{j} \bar{D}_{3}$ one can also get $b b j$ and $t t j$ final states, opening the possibility to search for rare multi-top events and events with same sign top pairs.

Overall, signals from direct RPV chargino decays complement previous studies, and looking for them is necessary in order to make a complete search for supersymmetry at the LHC. Moreover, since these signatures are directly correlated to the flavour and group structure of the theory, which determines the particle mass correlations and level of unification at high scales, it is hoped that any observable signal will help to distinguish between different possible realisations of supersymmetry.

\section{Acknowledgments}

The CPU intensive parts of this work was performed on the Abel Cluster, owned by the University of Oslo and the Norwegian metacenter for High Performance Computing (NOTUR), and operated by the Research Computing Services group at USIT, the University of Oslo IT-department. The computing time was given by NOTUR allocation NN9284K, financed through the Research Council of Norway. N.-E. Bomark is funded in part by the Welcome Programme of the Foundation for Polish Science. The use of the CIS computer cluster at the National Centre for Nuclear Research is gratefully acknowledged. 
Open Access. This article is distributed under the terms of the Creative Commons Attribution License (CC-BY 4.0), which permits any use, distribution and reproduction in any medium, provided the original author(s) and source are credited.

\section{References}

[1] R. Barbier et al., R-parity violating supersymmetry, Phys. Rept. 420 (2005) 1 [hep-ph/0406039] [INSPIRE].

[2] G.D. Kribs, A. Martin and T.S. Roy, Supersymmetry with a Chargino NLSP and Gravitino LSP, JHEP 01 (2009) 023 [arXiv:0807.4936] [INSPIRE].

[3] L. Randall and R. Sundrum, Out of this world supersymmetry breaking, Nucl. Phys. B 557 (1999) 79 [hep-th/9810155] [INSPIRE].

[4] G.F. Giudice, M.A. Luty, H. Murayama and R. Rattazzi, Gaugino mass without singlets, JHEP 12 (1998) 027 [hep-ph/9810442] [INSPIRE].

[5] C. Brust, A. Katz, S. Lawrence and R. Sundrum, SUSY, the Third Generation and the LHC, JHEP 03 (2012) 103 [arXiv:1110.6670] [INSPIRE].

[6] M. Papucci, J.T. Ruderman and A. Weiler, Natural SUSY Endures, JHEP 09 (2012) 035 [arXiv:1110.6926] [INSPIRE].

[7] N.-E. Bomark, A. Kvellestad, S. Lola, P. Osland and A.R. Raklev, Long lived charginos in Natural SUSY?, JHEP 05 (2014) 007 [arXiv: 1310.2788] [INSPIRE].

[8] C.H. Chen, M. Drees and J.F. Gunion, A nonstandard string/SUSY scenario and its phenomenological implications, Phys. Rev. D 55 (1997) 330 [Erratum ibid. D 60 (1999) 039901] [hep-ph/9607421] [INSPIRE].

[9] J.L. Feng, T. Moroi, L. Randall, M. Strassler and S.-f. Su, Discovering supersymmetry at the Tevatron in wino LSP scenarios, Phys. Rev. Lett. 83 (1999) 1731 [hep-ph/9904250] [INSPIRE].

[10] T. Gherghetta, G.F. Giudice and J.D. Wells, Phenomenological consequences of supersymmetry with anomaly induced masses, Nucl. Phys. B 559 (1999) 27 [hep-ph/9904378] [INSPIRE].

[11] F. de Campos, M.A. Diaz, O.J.P. Eboli, M.B. Magro, W. Porod and S. Skadhauge, CERN LHC signals for neutrino mass model in bilinear R-parity violating mAMSB, Phys. Rev. D 77 (2008) 115025 [arXiv: 0803.4405] [INSPIRE].

[12] H.K. Dreiner, S. Lola and P. Morawitz, Chargino pair production at LEP-2 with broken R-parity: 4 jet final states, Phys. Lett. B 389 (1996) 62 [hep-ph/9606364] [INSPIRE].

[13] F. Takayama and M. Yamaguchi, Gravitino dark matter without R-parity, Phys. Lett. B 485 (2000) 388 [hep-ph/0005214] [INSPIRE].

[14] W. Buchmüller, L. Covi, K. Hamaguchi, A. Ibarra and T. Yanagida, Gravitino Dark Matter in R-Parity Breaking Vacua, JHEP 03 (2007) 037 [hep-ph/0702184] [INSPIRE].

[15] S. Lola, P. Osland and A.R. Raklev, Radiative gravitino decays from R-parity violation, Phys. Lett. B 656 (2007) 83 [arXiv:0707.2510] [INSPIRE].

[16] ATLAS collaboration, Observation of a new particle in the search for the Standard Model Higgs boson with the ATLAS detector at the LHC, Phys. Lett. B 716 (2012) 1 [arXiv:1207.7214] [INSPIRE]. 
[17] CMS collaboration, Observation of a new boson at a mass of $125 \mathrm{GeV}$ with the CMS experiment at the LHC, Phys. Lett. B $\mathbf{7 1 6}$ (2012) 30 [arXiv:1207.7235] [INSPIRE].

[18] F. Feroz and M.P. Hobson, Multimodal nested sampling: an efficient and robust alternative to MCMC methods for astronomical data analysis,

Mon. Not. Roy. Astron. Soc. 384 (2008) 449 [arXiv:0704.3704] [INSPIRE].

[19] F. Feroz, M.P. Hobson and M. Bridges, MultiNest: an efficient and robust Bayesian inference tool for cosmology and particle physics, Mon. Not. Roy. Astron. Soc. 398 (2009) 1601 [arXiv: 0809.3437] [INSPIRE].

[20] J.R. Ellis, K. Enqvist, D.V. Nanopoulos and K. Tamvakis, Gaugino Masses and Grand Unification, Phys. Lett. B 155 (1985) 381 [inSPIRE].

[21] S.P. Martin, Non-universal gaugino masses from non-singlet F-terms in non-minimal unified models, Phys. Rev. D 79 (2009) 095019 [arXiv:0903.3568] [INSPIRE].

[22] T.-F. Feng, L. Sun and X.-Y. Yang, Electroweak and supersymmetric two-loop corrections to lepton anomalous magnetic and electric dipole moments, Nucl. Phys. B 800 (2008) 221 [arXiv:0805.1122] [INSPIRE].

[23] J.R. Ellis, J.S. Lee and A. Pilaftsis, Electric Dipole Moments in the MSSM Reloaded, JHEP 10 (2008) 049 [arXiv:0808.1819] [INSPIRE].

[24] K. Cheung, O.C.W. Kong and J.S. Lee, Electric and anomalous magnetic dipole moments of the muon in the MSSM, JHEP 06 (2009) 020 [arXiv:0904.4352] [INSPIRE].

[25] W. Altmannshofer, A.J. Buras, S. Gori, P. Paradisi and D.M. Straub, Anatomy and Phenomenology of FCNC and CPV Effects in SUSY Theories, Nucl. Phys. B 830 (2010) 17 [arXiv:0909.1333] [INSPIRE].

[26] H.-C. Cheng, B.A. Dobrescu and K.T. Matchev, Generic and chiral extensions of the supersymmetric standard model, Nucl. Phys. B 543 (1999) 47 [hep-ph/9811316] [InSPIRE].

[27] G.F. Giudice and A. Pomarol, Mass degeneracy of the Higgsinos, Phys. Lett. B 372 (1996) 253 [hep-ph/9512337] [InSPIRE].

[28] M.E. Cabrera, J.A. Casas and R. Ruiz de Austri, Bayesian approach and Naturalness in MSSM analyses for the LHC, JHEP 03 (2009) 075 [arXiv: 0812.0536] [INSPIRE].

[29] CMS collaboration, Top mass combination, CMS-PAS-TOP-11-018.

[30] Particle Data Group collaboration, J. Beringer et al., Review of Particle Physics (RPP), Phys. Rev. D 86 (2012) 010001 [inSPIRE].

[31] B.C. Allanach, SOFTSUSY: a program for calculating supersymmetric spectra, Comput. Phys. Commun. 143 (2002) 305 [hep-ph/0104145] [INSPIRE].

[32] S. Heinemeyer, W. Hollik and G. Weiglein, FeynHiggs: A program for the calculation of the masses of the neutral CP even Higgs bosons in the MSSM,

Comput. Phys. Commun. 124 (2000) 76 [hep-ph/9812320] [INSPIRE].

[33] S. Heinemeyer, W. Hollik and G. Weiglein, The Masses of the neutral CP-even Higgs bosons in the MSSM: Accurate analysis at the two loop level, Eur. Phys. J. C 9 (1999) 343 [hep-ph/9812472] [INSPIRE].

[34] G. Degrassi, S. Heinemeyer, W. Hollik, P. Slavich and G. Weiglein, Towards high precision predictions for the MSSM Higgs sector, Eur. Phys. J. C 28 (2003) 133 [hep-ph/0212020] [INSPIRE]. 
[35] M. Frank, T. Hahn, S. Heinemeyer, W. Hollik, H. Rzehak and G. Weiglein, The Higgs Boson Masses and Mixings of the Complex MSSM in the Feynman-Diagrammatic Approach, JHEP 02 (2007) 047 [hep-ph/0611326] [INSPIRE].

[36] G. Bélanger, F. Boudjema, A. Pukhov and A. Semenov, MicrOMEGAs: A program for calculating the relic density in the MSSM, Comput. Phys. Commun. 149 (2002) 103 [hep-ph/0112278] [INSPIRE].

[37] G. Bélanger, F. Boudjema, A. Pukhov and A. Semenov, MicrOMEGAs: Version 1.3, Comput. Phys. Commun. 174 (2006) 577 [hep-ph/0405253] [INSPIRE].

[38] G. Bélanger et al., Indirect search for dark matter with MicrOMEGAs2.4, Comput. Phys. Commun. 182 (2011) 842 [arXiv:1004.1092] [INSPIRE].

[39] CMS collaboration, Measurement of the $B_{s}^{0} \rightarrow \mu^{+} \mu^{-}$branching fraction and search for $B^{0} \rightarrow \mu^{+} \mu^{-}$with the CMS Experiment, Phys. Rev. Lett. 111 (2013) 101804 [arXiv: 1307.5025] [INSPIRE].

[40] LHCb collaboration, Measurement of the $B_{s}^{0} \rightarrow \mu^{+} \mu^{-}$branching fraction and search for $B^{0} \rightarrow \mu^{+} \mu^{-}$decays at the LHCb experiment, Phys. Rev. Lett. 111 (2013) 101805 [arXiv: 1307.5024] [INSPIRE].

[41] LHCb collaboration, Measurement of the $B_{s}^{0} \rightarrow \mu^{+} \mu^{-}$branching fraction and search for $B^{0} \rightarrow \mu^{+} \mu^{-}$decays at the LHCb experiment, LHCB-PAPER-2013-046 (2013), supplementary material.

[42] CDF, D0 collaborations, T.A. Aaltonen et al., Combination of CDF and D0 W-Boson Mass Measurements, Phys. Rev. D 88 (2013) 052018 [arXiv: 1307.7627] [InSPIRE].

[43] K. Hagiwara, R. Liao, A.D. Martin, D. Nomura and T. Teubner, $(g-2)_{\mu}$ and $\alpha\left(M_{Z}^{2}\right)$ re-evaluated using new precise data, J. Phys. G 38 (2011) 085003 [arXiv:1105.3149] [INSPIRE].

[44] C. Gnendiger, D. Stöckinger and H. Stöckinger-Kim, The electroweak contributions to $(g-2)_{\mu}$ after the Higgs boson mass measurement, Phys. Rev. D 88 (2013) 053005 [arXiv: 1306.5546] [INSPIRE].

[45] Heavy Flavor Averaging Group collaboration, Y. Amhis et al., Averages of B-Hadron, C-Hadron and tau-lepton properties as of early 2012, arXiv:1207.1158 [INSPIRE].

[46] CMS collaboration, Precise determination of the mass of the Higgs boson and studies of the compatibility of its couplings with the standard model, CMS-PAS-HIG-14-009.

[47] ALEPH collaboration, D. Decamp et al., Searches for new particles in $Z$ decays using the ALEPH detector, Phys. Rept. 216 (1992) 253 [INSPIRE].

[48] R.R. de Austri, R. Trotta and L. Roszkowski, A Markov chain Monte Carlo analysis of the CMSSM, JHEP 05 (2006) 002 [hep-ph/0602028] [INSPIRE].

[49] R. Trotta, F. Feroz, M.P. Hobson, L. Roszkowski and R.R. de Austri, The Impact of priors and observables on parameter inferences in the Constrained MSSM, JHEP 12 (2008) 024 [arXiv: 0809.3792] [INSPIRE].

[50] P. Gondolo, J. Edsjo, P. Ullio, L. Bergstrom, M. Schelke and E.A. Baltz, DarkSUSY: Computing supersymmetric dark matter properties numerically, JCAP 07 (2004) 008 [astro-ph/0406204] [INSPIRE].

[51] P. Richardson, Simulations of R-parity violating SUSY models, hep-ph/0101105 [INSPIRE].

[52] V.D. Barger, G.F. Giudice and T. Han, Some New Aspects of Supersymmetry R-Parity Violating Interactions, Phys. Rev. D 40 (1989) 2987 [INSPIRE]. 
[53] B.C. Allanach, A. Dedes and H.K. Dreiner, Bounds on R-parity violating couplings at the weak scale and at the GUT scale, Phys. Rev. D 60 (1999) 075014 [hep-ph/9906209] [INSPIRE].

[54] A.J. Barr, C.G. Lester, M.A. Parker, B.C. Allanach and P. Richardson, Discovering anomaly mediated supersymmetry at the LHC, JHEP 03 (2003) 045 [hep-ph/0208214] [INSPIRE].

[55] ATLAS collaboration, Search for charginos nearly mass-degenerate with the lightest neutralino based on a disappearing-track signature in pp collisions at $\sqrt{s}=8 \mathrm{TeV}$ with the ATLAS detector, ATLAS-CONF-2013-069 (2013).

[56] T. Sjöstrand, S. Mrenna and P.Z. Skands, PYTHIA 6.4 Physics and Manual, JHEP 05 (2006) 026 [hep-ph/0603175] [INSPIRE].

[57] T. Sjöstrand, S. Mrenna and P.Z. Skands, A Brief Introduction to PYTHIA 8.1, Comput. Phys. Commun. 178 (2008) 852 [arXiv:0710.3820] [InSPIRE].

[58] M. Cacciari and G.P. Salam, Dispelling the $N^{3}$ myth for the $k_{t}$ jet-finder, Phys. Lett. B 641 (2006) 57 [hep-ph/0512210] [INSPIRE].

[59] S. Catani, Y.L. Dokshitzer, M. Olsson, G. Turnock and B.R. Webber, New clustering algorithm for multi-jet cross-sections in $e^{+} e^{-}$annihilation, Phys. Lett. B 269 (1991) 432 [INSPIRE].

[60] S. Catani, Y.L. Dokshitzer, M.H. Seymour and B.R. Webber, Longitudinally invariant $K_{t}$ clustering algorithms for hadron hadron collisions, Nucl. Phys. B 406 (1993) 187 [INSPIRE].

[61] N.-E. Bomark, D. Choudhury, S. Lola and P. Osland, Flavour Structure of R-violating Neutralino Decays at the LHC, JHEP 07 (2011) 070 [arXiv:1105.4022] [INSPIRE].

[62] ATLAS collaboration, Search for Supersymmetry at the high luminosity LHC with the ATLAS experiment, ATL-COM-PHYS-2014-555 (2014).

[63] J.M. Campbell, R.K. Ellis and C. Williams, Vector boson pair production at the LHC, JHEP 07 (2011) 018 [arXiv:1105.0020] [INSPIRE].

[64] W. Beenakker, M. Klasen, M. Krämer, T. Plehn, M. Spira and P.M. Zerwas, The production of charginos/neutralinos and sleptons at hadron colliders, Phys. Rev. Lett. 83 (1999) 3780 [Erratum ibid. 100 (2008) 029901] [hep-ph/9906298] [INSPIRE].

[65] ATLAS collaboration, Search for supersymmetry in events with four or more leptons in $\sqrt{s}=8 \mathrm{TeV}$ pp collisions with the ATLAS detector, Phys. Rev. D 90 (2014) 052001 [arXiv: 1405.5086] [INSPIRE].

[66] B. Allanach, S. Biswas, S. Mondal and M. Mitra, Explaining a CMS eejj Excess With $\mathcal{R}$-parity Violating Supersymmetry and Implications for Neutrinoless Double Beta Decay, arXiv: 1408.5439 [INSPIRE].

[67] CMS collaboration, Search for heavy neutrinos and $W$ bosons with right-handed couplings in proton-proton collisions at $\sqrt{s}=8$ TeV, Eur. Phys. J. C 74 (2014) 3149 [arXiv:1407.3683] [INSPIRE].

[68] J.M. Butterworth, J.R. Ellis, A.R. Raklev and G.P. Salam, Discovering baryon-number violating neutralino decays at the LHC, Phys. Rev. Lett. 103 (2009) 241803 [arXiv:0906.0728] [INSPIRE].

[69] CMS collaboration, Searches for light- and heavy-flavour three-jet resonances in pp collisions at $\sqrt{s}=8 \mathrm{TeV}$, Phys. Lett. B 730 (2014) 193 [arXiv:1311.1799] [INSPIRE]. 